\title{
Experimental Demonstration of the Potential 5G Based Multiplexed Radio Frequency Signals Transmission in Passive Optical Network
}

\author{
Hum Nath PARAJULI ${ }^{1,2}$, Eszter UDVARY ${ }^{2}$, Julien POETTE ${ }^{3}$ \\ ${ }^{1}$ Scuola Superiore Sant Anna, TeCIP Institute, via Giuseppe Moruzzi, 1, 56124, Pisa, Italy \\ ${ }^{2}$ Dept. of Broadband Info-Communication and Electromagnetic Theory, Budapest University of Technology and \\ Economics, 3 Muegyetem rakpart, 1111 Budapest, Hungary \\ ${ }^{3}$ Universite Grenoble Alpes, CNRS, Institute of Engineering, IMEP-LAHC, 38000, Grenoble, France \\ humnath.parajuli@santannapisa.it,udvary@hvt.bme.hu,julien.poette@imep.grenoble-inp.fr
}

Submitted January 12, 2020 / Accepted June 15, 2020

\begin{abstract}
Future passive optical network (PON) designs with $5 G$ have been expected to provide simultaneous multiple wireless signals to the end-users. This paper proposes and experimentally demonstrates the simultaneous transmission of baseband 4-pulse amplitude modulation (4-PAM), radio frequency (RF) filter bank multicarrier (FBMC), and universal filter orthogonal frequency division multiplexing (UF-OFDM) signals in a PON. A single optical wavelength from a laser source is used to demonstrate the signal transmission over a PON. In contrast with conventional on-off keying (OOK) modulation format, 4-PAM modulation format can provide double bandwidth efficiency. Due to the property of high suppression for out of band emission, UF-OFDM and FBMC are considered as potential $5 G$ modulation formats. In the optical line terminal (OLT), the composite signal consisting of 4-PAM, FBMC, and UF-OFDM is designed, generated, and transmitted using optical intensity modulation. The received signal is extracted in the optical network unit (ONU) and demodulated using digital signal processing techniques. Each of the modulation formats mentioned above is designed with a 2 Gbps data rate constituting a total of 6 Gbps aggregate data rate. The bit error rate (BER) and error vector magnitude (EVM) values have been measured after $25 \mathrm{~km}$ fiber length. Measurement results show EVM values below $12.5 \%$ as a figure of merit as proposed by 3 GPP LTE for 16 QAM modulation.
\end{abstract}

\section{Keywords}

5G, FBMC, passive optical network, radio over fiber, UF-OFDM, wired-wireless convergence

\section{Introduction}

The passive optical network (PON) uses a fixed access network, which can provide flexibility in high bandwidth signal transmission. The PON system is considered as a support infrastructure for fronthauling and backhauling of $5 \mathrm{G}$ signals [1-3]. Future $5 \mathrm{G}$ networks should be capable of handling multi-signals/services in the transport mediums to keep compatibility with the current legacy wireless services. Thus, it is essential to explore and evaluate the performance of the multiplexed potential $5 \mathrm{G}$ wireless signals transmission through PON. It is expected that future $5 \mathrm{G}$ networks should provide $1-10$ Gbps wireless access to the end-users $[2,4,5]$, due to which spectral efficient modulation formats have to be used.

Multicarrier modulation formats, such as orthogonal frequency division multiplexing (OFDM), have been widely used in contemporary $4 \mathrm{G}$ systems to increase spectral efficiency. OFDM is also considered a robust modulation format against propagation impairments over optical fiber such as chromatic dispersion (CD) [6], [7]. However, OFDM requires a cyclic prefix $(\mathrm{CP})$, which reduces the spectral efficiency. OFDM uses a rectangular window in each subcarrier that causes large out of band emission, thus requiring large guard bands in multi-sub-bands systems. These limitations can be overcome with advanced multicarrier modulation formats. In this paper, filter bank multicarrier (FBMC) and universal filter orthogonal frequency division multiplexing (UF-OFDM) methods are applied. Typical OFDM side-lobe suppression ratio is about $13 \mathrm{~dB}$, whereas FBMC signals can be designed with the side-lobe suppression ratio as high as $40 \mathrm{~dB}$ [8], [9]. In FBMC, due to the significant reduction of out of band emission and the combination of the filter banks and offset quadrature amplitude (OQAM), no CP is required [8-13].

FBMC demodulation scheme needs a filter for each subcarrier, resulting in increased complexity. In UF-OFDM, the total bandwidth is divided into several smaller subbands; each sub-band is then separately modulated using OFDM and filtered. This technique reduces the complexity in comparison with FBMC. UF-OFDM can use quadrature amplitude (QAM) modulation, but FBMC requires conversion of QAM to OQAM to maintain orthogonal sub-bands, which finally doubles the sampling rate [13-16]. 4-pulse 
amplitude modulation (4-PAM) is compatible with optical intensity modulation and direct detection (IM/DD) systems, but also multiplies by 2 the bandwidth efficiency compared to the traditional on-off keying (OOK) modulation. Due to this benefit, huge research interests are recently shown on 4-PAM for cost-effective optical access network design [17-19].

The experimental demonstration of OFDM and FBMC based PON is given in [20]. To investigate aggregate bandwidths of less than $1.5 \mathrm{GHz}$, the performance comparison of OFDM and FBMC signals at mm-wave radio over fiber (RoF) was recently studied [9], [11]. In these demonstrations, FBMC shows superior performance than OFDM. Adaptively modulated FBMC used in a wired-wireless converged optical network with the aggregate signal bandwidth of $1.5 \mathrm{GHz}$ was demonstrated in [21]. Recently, the convergence of 4-PAM as a wired signal and UF-OFDM and generalized filter multicarrier (GFDM) used for a wireless signal in a PON has been demonstrated [22]. Above mentioned demonstrations of the multiplexed signal transmission in a PON have not been dealt with the evaluation of potential $5 \mathrm{G}$ modulation formats such as FBMC, UF-OFDM, and 4-PAM. Also, the demonstration of [22] is realized using a very low wireless signal bandwidth of $1.95 \mathrm{MHz}$ and hence for very low data rate. Previously, we have demonstrated the multiplexed multi-sub-bands FBMC and 4-PAM transmission in PON using numerical simulation [23]. The study proposed in this communication demonstrates the experimental validation of the multiplexed 4-PAM, FBMC, and UF-OFDM signals simultaneously transmitted in PON. In order to transmit each signal at a bit rate of $2 \mathrm{GBit} / \mathrm{s}$, the bandwidth of the designed FBMC signal is $1 \mathrm{GHz}$, UF-OFDM is $500 \mathrm{MHz}$, and 4-PAM is $1 \mathrm{GHz}$. The aggregation of the 3 sub-bands modulated with different formats results in a total data rate of 6 Gbps. The 4-PAM, UF-OFDM, and FBMC bands are extracted and demodulated in the receiver by using digital signal processing (DSP) techniques. The performance of the multiplexed signal has been evaluated using bit error rate $(\mathrm{BER})$ and error vector magnitude (EVM) measurements.

The organization of this paper is as follows: in Sec. 2, 4-PAM, FBMC, and UF-OFDM signals generation methods are given. In Sec. 3, the description of the model of the experimental optical setup is given. Section 4 presents the signal processing techniques for the extraction and demodulation of the received multiplexed signals. Section 5 illustrates and discusses the measurement results. Finally, Section 6 concludes the paper.

\section{Multiplexed Signal Generation}

The MATLAB program is used to develop the routines for generating offline 4-PAM, FBMC, and UF-OFDM signals. The baseband 4-PAM signal is generated with $1 \mathrm{GHz}$ bandwidth. The total number of bits used to create the 4-PAM signal is 32768. The sampling frequency is 12 GS/s. Each 4-PAM symbol is up-sampled with 6 samples

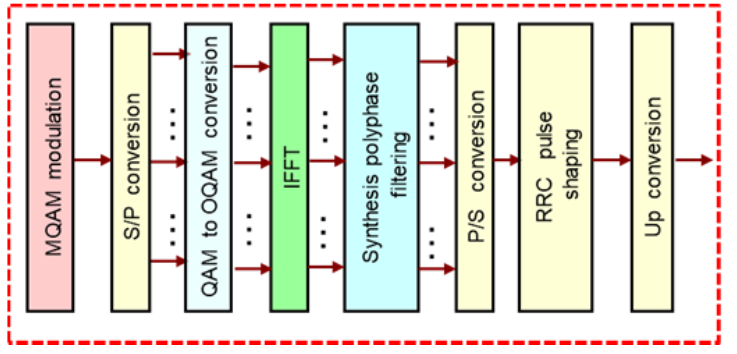

Fig. 1. Functional block diagram of FBMC signal generation.

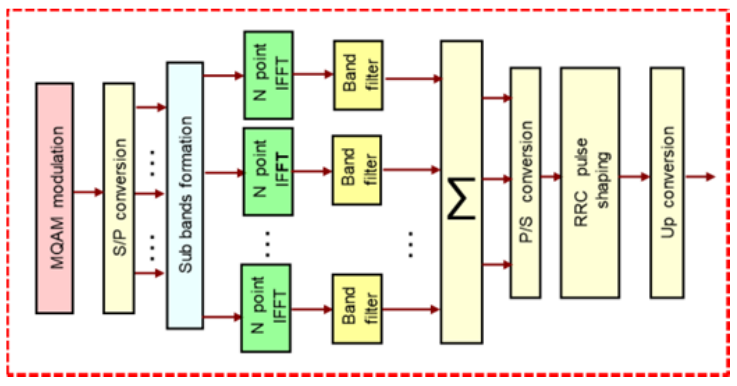

Fig. 2. Functional block diagram of UF-OFDM signal generation.

For the pulse shaping, a root raised cosine (RRC) filter with a roll-off factor of 0.2 is used.

The simplified DSP block diagram for generating the FBMC signal is shown in Fig. 1. The total number of bits used is 16384. First, the input bit-stream is mapped into M-QAM symbols, and then it is converted from serial to parallel (S/P) streams. After this, the QAM to offset QAM (OQAM) conversion is performed [12], [13]. Then, IFFT of size 1024 is applied. Each subcarrier is filtered with a well-designed prototype filter; this process is called synthesis poly-phase filtering (SPF) [12]. After this, parallel to serial conversion $(\mathrm{P} / \mathrm{S})$ method is applied, and then the RRC filter with a roll-off factor of 0.2 is used to optimize the signal to noise ratio (SNR). The sampling frequency is fixed to $12 \mathrm{GS} / \mathrm{s}$, and each OQAM symbol is up-sampled with 12 samples. 4 FBMC symbols are created, which form a bandwidth of $1 \mathrm{GHz}$. The designed baseband FBMC signal is up-converted to a radio frequency signal (RF) with a carrier frequency of $2.2 \mathrm{GHz}$.

Figure 2 shows the simplified DSP block diagram for generating the UF-OFDM signal [14-16]. The total number of bits used is 12288. First, the bit to symbol mapping is performed, and then it is converted from serial to parallel (S/P) streams. After this, the sub-band size is defined, subband mapping is performed, and then IFFT of size 1024 is applied. The sampling rate is $12 \mathrm{GS} / \mathrm{s}$, and each QAM symbol is up-sampled with 12 samples for each QAM symbol. 32 QAM symbols form one UF-OFDM sub-band. The total numbers of UF-OFDM sub-bands are 32. Each sub-band is filtered with a well-designed filter to suppress the out-of-band emission. In our case, we employed a Chebyshev window-based filter. Each sub-band is added to constitute the composite UF-OFDM signal. After this, P/S conversion is performed, and then, RRC filter with a rolloff factor of 0.2 is applied to optimize the SNR. The bandwidth of the generated signal is $500 \mathrm{MHz}$. The baseband 


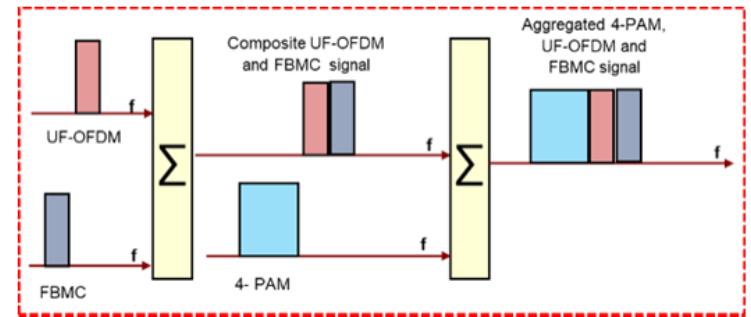

Fig. 3. Multiplexing of the 4-PAM, FBMC, and UF-OFDM signals.

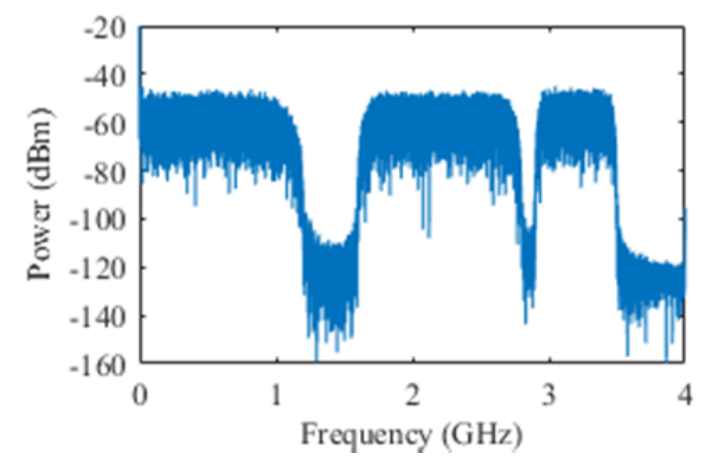

Fig. 4. Generated spectra of the multiplexed 4-PAM, UF-OFDM, and FBMC signal (offline).

UF-OFDM signal is upconverted to a $3.2 \mathrm{GHz}$ carrier frequency.

The 4-PAM signal is added up with the UF-OFDM and the FBMC signals to generate a multiplexed composite signal, as shown in Fig. 3. After this, signal normalization and clipping are applied to maintain the peak-to-peak voltage amplitude at $1 \mathrm{~V}$. The frequency gap between the 4-PAM and FBMC is $300 \mathrm{MHz}$ and between FBMC and UF-OFDM is $150 \mathrm{MHz}$. These frequency gaps are taken after observing a series of BER performance analysis in experiments having different frequency gaps. The total bandwidth of the aggregated 4-PAM, FBMC, and UFOFDM signals from dc is $3.65 \mathrm{GHz}$. The generated offline code output is uploaded to an arbitrary waveform generator (AWG). Figure 4 shows the offline generated spectra of the multiplexed 4-PAM, FBMC, and UF-OFDM signal.

\section{Optical System Experiment}

Figure 5 represents the simplified block diagram of the optical transmitter and receiver for the proposed PON setup. The setup consists of an optical line terminal (OLT) and an optical network unit (ONU) connected through an optical fiber.

In the OLT, a distributed feedback (DFB) laser (Mitsubishi, FU-68PDF-5M63A) at $1551.82 \mathrm{~nm}$ wavelength with an optical linewidth of $1 \mathrm{MHz}$ is used. The measured laser output power is about $17 \mathrm{dBm}$. The used intensity modulator (IM) (Sumitomo Osaka Cement Co. Ltd., T-DEH1.5-20-ADC) has a half-wave voltage of $8 \mathrm{~V}$ and insertion loss of $6 \mathrm{~dB}$. The IM is driven by a multiplexed composite signal generated from the AWG (Tektronics, AWG 7122B) and biased in a linear transmission

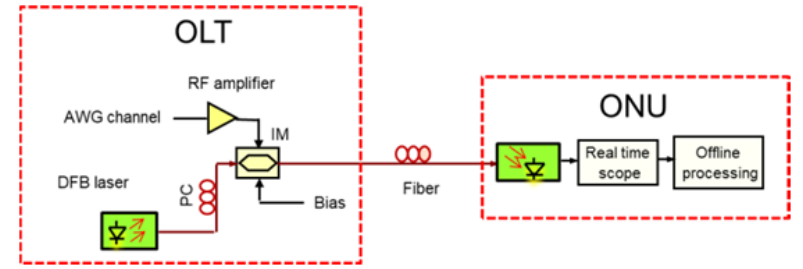

Fig. 5. Block diagram of the PON setup with OLT and ONU.

point. The optical power at the IM input is optimized with a polarization controller (PC). The peak-to-peak amplitude from AWG is $500 \mathrm{mV}$, and the sampling rate is $12 \mathrm{GS} / \mathrm{s}$. The AWG channel output is amplified with a radio frequency (RF) amplifier (Miteq LNA AFS42-00101800-2510P-42) before applying to the modulator. The RF amplifier has a gain of $25 \mathrm{~dB}$. The used optical fiber is a standard single-mode fiber (SMF-28) of $25 \mathrm{~km}$ length. In the ONU, a PIN photodiode (Finisar XPDV3120R $70 \mathrm{GHz}$ photodetector) is applied. The photodetector detected the optical signal with $0.6 \mathrm{~A} / \mathrm{W}$ responsivity at $1550 \mathrm{~nm}$. The received signal is recorded with a real-time scope (RTS) (Agilent, 54855A Infiniium Oscilloscope) of $20 \mathrm{GS} / \mathrm{s}$ for further offline DSP using the developed MATLAB code.

\section{Signal Processing in Receiver}

After the photodetection, the received signal is captured in ONU with real-time scope (RTS) at $20 \mathrm{GS} / \mathrm{s}$. An example of the measured spectrum is shown in Fig. 6.

The 4-PAM, UF-OFDM, and FBMC signals are extracted and demodulated separately. The transmitter and receiver symbol alignment is achieved with the cross-correlation technique [24]. For the FBMC, the signal at baseband is obtained after downconverting it with the intermediate frequency (IF) of $2.2 \mathrm{GHz}$ along with the RRC low pass filter of roll-off factor 0.2 as in the transmitter side. After this, the resampling is applied to downsample the signal at two samples for each OQAM symbols.

Figure 7 represents the steps of FBMC decoding steps, as explained in [9], [12], [13]. The decoding steps involve S/P conversion, O-QAM to QAM conversion, and $\mathrm{P} / \mathrm{S}$ conversion. The channel response is determined through the first 16 QAM symbols as a training signal to equalize the FBMC signal. Furthermore, to optimize the equalization process, a simple one-tap recursive least square (RLS) equalizer with a forgetting factor of 0.9 is used. The BER and EVM measurements are performed after the equalization process.

The 4-PAM signal extraction and demodulation steps are described in Fig. 8(a). Figure 8(b) shows the extracted two sidebands of the 4-PAM signal after using a square bandpass filter at center frequency $0 \mathrm{~Hz}$ and a bandwidth of $2.4 \mathrm{GHz}$. The equalization of 4-PAM signal has been performed with 11 taps adaptive feedforward finite impulse response (FFE) equalizer with least mean square (LMS) adaptation. For the LMS algorithm, the first 64 samples of the 4-PAM signal are used as a training signal. The BER and EVM performances are measured after the equalization. 


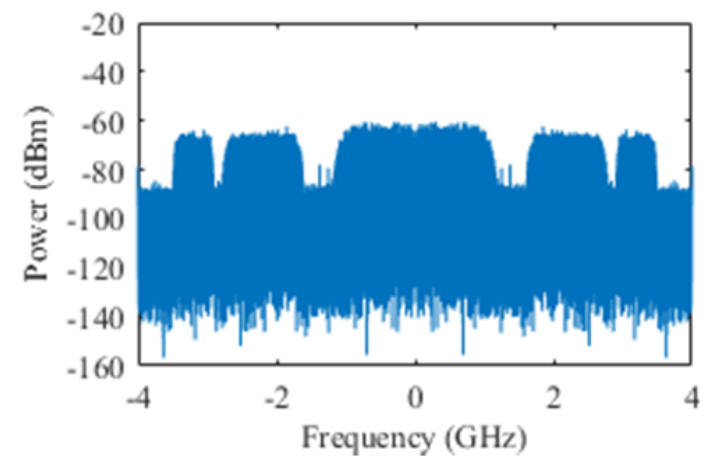

Fig. 6. The spectrum of the signal at the output of the photodetector in the ONU, applying $25 \mathrm{~km}$ fiber length.

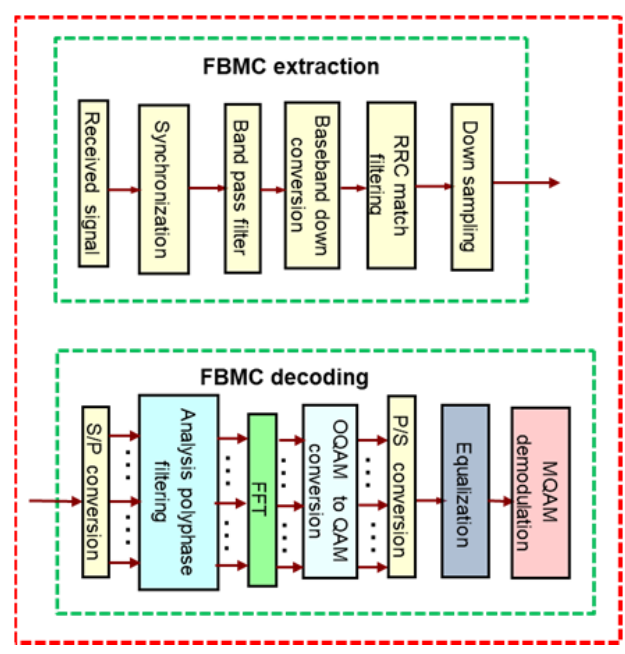

Fig. 7. FBMC sub-bands extraction and decoding.

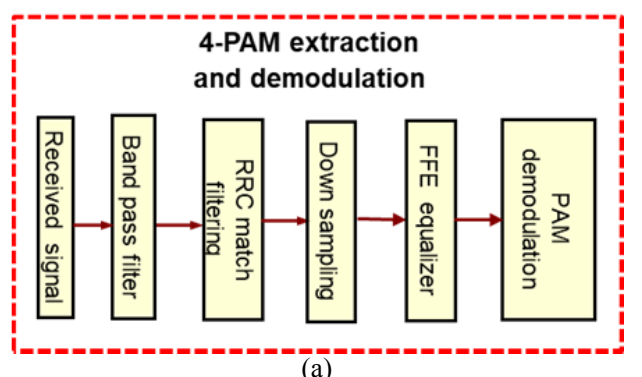

(a)

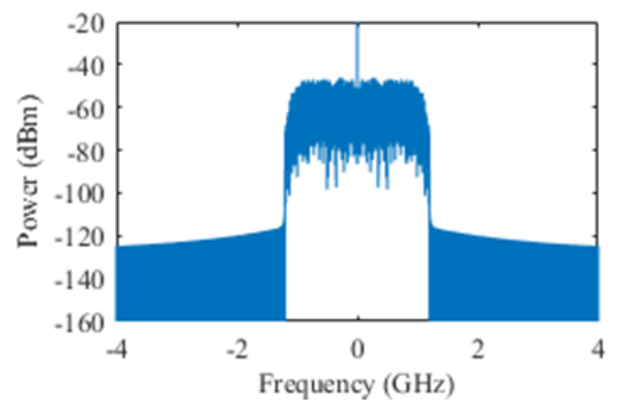

(b)

Fig. 8. Extraction and demodulation of 4-PAM signal.

Baseband UF-OFDM signal is extracted by downconverting it with an IF frequency of $3.2 \mathrm{GHz}$, the same that was used in the signal generation. Then, the RRC low pass filter of a roll-off factor of 0.2 is applied as in the transmitter

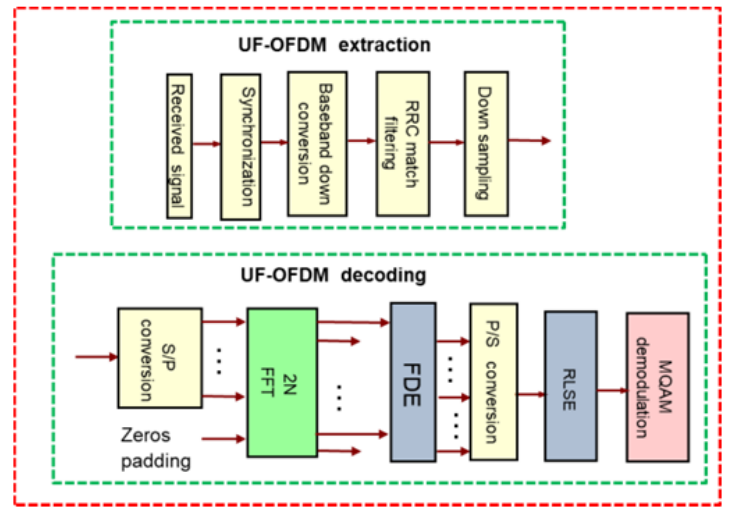

Fig. 9. UF-OFDM signal extraction and decoding.

side. Then, the signal is resampled at two samples per QAM symbol. The UF-OFDM decoding routines are finally implemented, as shown in [14-16].

First, the serial stream is converted into a parallel stream $(\mathrm{S} / \mathrm{P})$. After this, $2 \mathrm{~N}$ points $\mathrm{FFT}$ is applied. The lower half of the inputs to the FFT is padded zeros due to which alternate subcarriers of FFT output correspond to the desired output. After this, each subcarrier is equalized with zero-forcing frequency domain equalizer to combat the effect of sub-band filtering and channel effect. Consequently, parallel to serial $(\mathrm{P} / \mathrm{S})$ conversion process is applied. The first 32 QAM samples are used as a training signal to calculate the channel response, which is used to equalize the signal. Furthermore, to optimize the equalization process, a simple one-tap RLS equalizer with a forgetting factor of 0.9 is used. Then, the performance of the signal is evaluated with BER and EVM measurements.

\section{Results and Discussions}

Chromatic dispersion (CD) and fiber attenuation are the main significant impairments for transmitting an RF signal through optical fibers. The $\mathrm{CD}$ causes periodic power variation of the received signal, which depends on the value of the RF signal frequency. From the theoretical calculation and numerical simulation, it can be shown that the first null power point occurs at $139 \mathrm{~km}$ for a $5 \mathrm{GHz} \mathrm{RF}$ signal propagating in a fiber having a standard dispersion of $18 \mathrm{ps} / \mathrm{nm} / \mathrm{km}$ dispersion at the laser wavelength of $1551.82 \mathrm{~nm}$ [13]. In the designed signal, the aggregated signal bandwidth from dc is $3.65 \mathrm{GHz}$ and used fiber length is $25 \mathrm{~km}$. Thus, the power fading effect in the received signal can be considered negligible. In this case, the primary transmission impairments are fiber attenuation and electronic noise. Electronic noise arises from electronic components such as AWG, RF amplifier, photodetector, RTS, etc., that affect the signal to noise ratio (SNR) of the received signal and thus the performance of the system. The received optical power (ROP) is attenuated using a variable optical power attenuator (VOA) and measured just before the photodetector to emulate the fiber attenuation. By considering the power losses in the optical link $(6 \mathrm{~dB}$ IM insertion loss, $3 \mathrm{~dB}$ IM quadrature point operation loss, $5 \mathrm{~dB}$ optical fiber loss for $25 \mathrm{~km}, 1 \mathrm{~dB}$ VOA insertion loss, 
$1 \mathrm{~dB}$ connectors/splicing losses), the received optical power before photodetector is about $0 \mathrm{dBm}$. The electrical SNR of the composite received signal at $0 \mathrm{dBm}$ ROP is about $20 \mathrm{~dB}$. The ROP of about $0 \mathrm{dBm}$ is already strong to consider for the practical situation in radio over fiber systems. Therefore extra optical amplification is not required. BER and EVM measurements are performed to study the performance of the system.

Figure 10 and Figure 11, respectively, show the BER and EVM values versus ROP. These results are obtained with the individual transmission of 4-PAM, FBMC, and UF-OFDM signals at the fiber length of $25 \mathrm{~km}$. It can be seen from the curves, FBMC shows better performance compared to 4-PAM and UF-OFDM. At ROP of $-12 \mathrm{dBm}$, the BER of $10^{-3}$ can be obtained for the case of FBMC, at $-11 \mathrm{dBm}$ for the case of UF-OFDM and $-9 \mathrm{dBm}$ for the case of 4-PAM. This BER can satisfy the standard forward error correction limit (FEC) [25]. This experiment achieved EVM below $12.5 \%$ as a figure of merit as proposed by 3GPP LTE for 16 QAM modulation at ROP of $-12 \mathrm{dBm}$ for the case of $\mathrm{FBMC}$, and at $-11 \mathrm{dBm}$ for the case of UF-OFDM [13].

The FBMC and UF-OFDM signals are now multiplexed, and the composite signal is transmitted. The FBMC and UFOFDM signals are extracted separately from the received composite signal using a square bandpass filter. The BER with ROP is evaluated, as shown in Fig. 12, after $25 \mathrm{~km}$ fiber length for the extracted FBMC and UF-OFDM. For lower values of ROP, both modulation formats show poor performance. For the ROP of $-11.5 \mathrm{dBm}$, the BER of

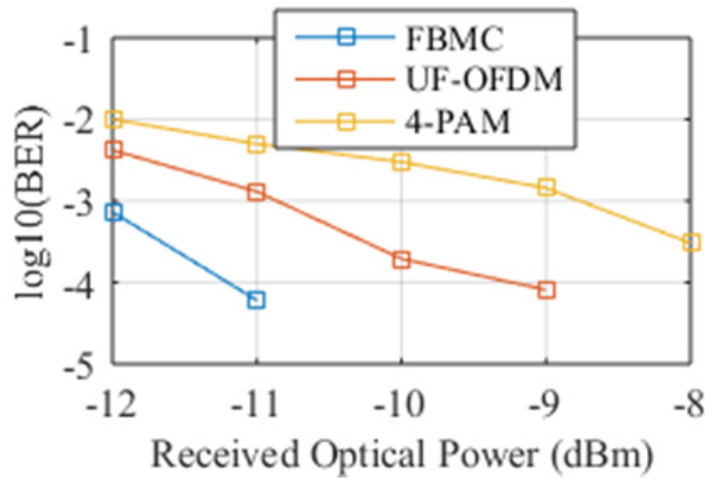

Fig. 10. BER comparisons for separate transmission.

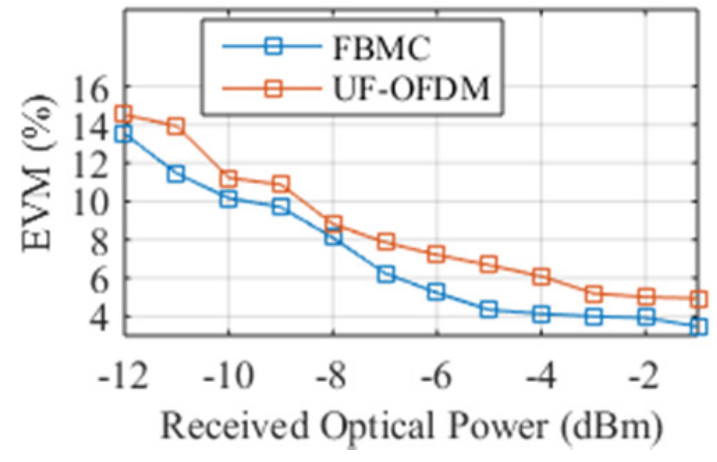

Fig. 11. EVM comparisons of FBMC and UF-OFDM for separate transmission.

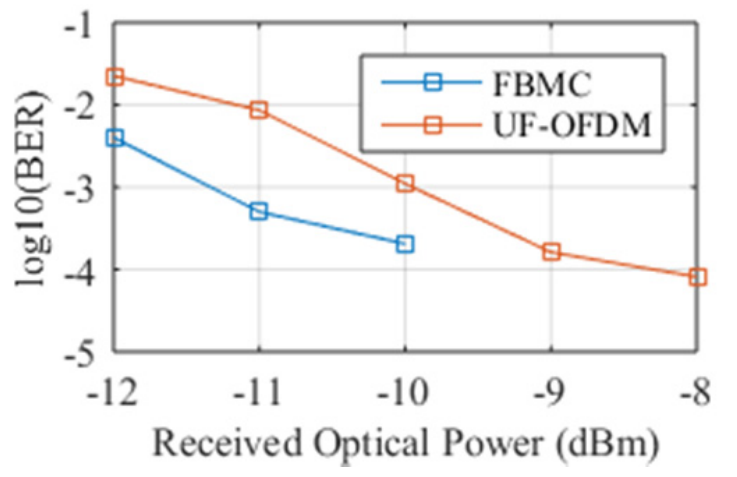

Fig. 12. BER comparisons of the multiplexed FBMC and UF-OFDM transmission.

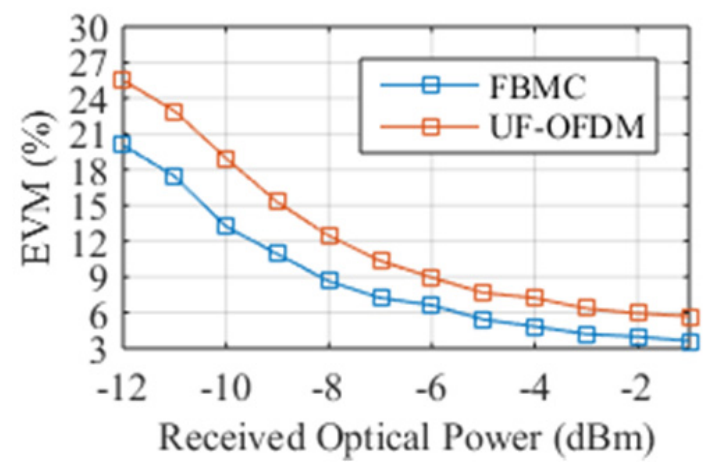

Fig. 13. EVM comparisons of the multiplexed FBMC and UF-OFDM transmission.

$10^{-3}$ can be obtained for the case of FBMC and at $-10 \mathrm{dBm}$ for the case of UF-OFDM.

Similarly, as shown in Fig. 13, the EVM of $12.5 \%$ is achieved at the ROP of $-10 \mathrm{dBm}$ and $-8 \mathrm{dBm}$ for FBMC and UF-OFDM cases, respectively. As the ROP increases, the FBMC signal shows better performance than the UFOFDM signal. For the two signal case, the performance is slightly limited in comparison with individual transmission case due to the fact of the signal mixing effect that leads to distortion.

The 4-PAM, FBMC, and UF-OFDM signals are now multiplexed, and the composite signal is transmitted over $25 \mathrm{~km}$ of optical fiber. The signals are extracted separately from the received composite signal using a square bandpass filter. The BER and EVM values with different ROP values are evaluated, as shown in Fig. 14 and Fig. 15. For lower values of ROP, all the modulation schemes show the poor performance. For the ROP of $-11.0 \mathrm{dBm}$, the BER of $10^{-3}$ can be obtained for FBMC, at $-9 \mathrm{dBm}$ for the UF-OFDM, and $-7 \mathrm{dBm}$ for 4-PAM.

Similarly, the EVM of $12.5 \%$ is achieved at the ROP of $-9 \mathrm{dBm}$ and $-5 \mathrm{dBm}$ for the FBMC and UF-OFDM cases, respectively. The constellation diagrams at the received optical power of $-1 \mathrm{dBm}$ for both FBMC and UFOFDM cases are also shown as insets in Fig. 15. As the ROP increases, the FBMC signal shows better performance than UF-OFDM and 4-PAM. The degradation of performance in this case in comparison with the previous results is due to the signal mixing effect. 


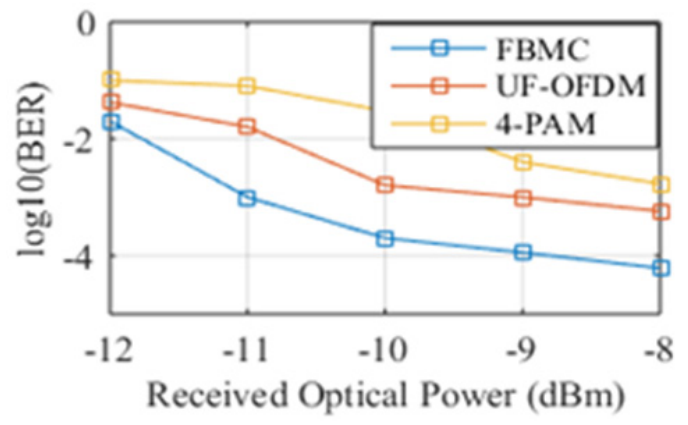

Fig. 14. BER comparisons of the multiplexed 4-PAM, UF-OFDM and FBMC transmission.

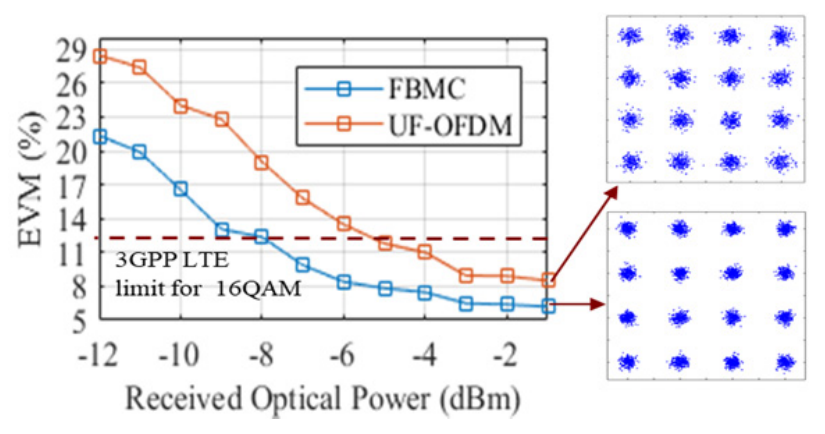

Fig. 15. EVM comparisons of the multiplexed 4-PAM, UF-OFDM and FBMC transmission.

The presented results are achieved with the addition of the simple RLS based post-equalizer in the receiver DSP for the FBMC and UF-OFDM performance optimization. Similarly, for 4-PAM, liner FFE is used.

\section{Conclusions}

In this paper, we demonstrated the performance of multiplexed RF signals transmission in PON using $2 \mathrm{Gbit} / \mathrm{s}$ signal modulated using FBMC of $1 \mathrm{GHz}$ bandwidth, UF-OFDM of $500 \mathrm{MHz}$ bandwidth, and 4-PAM of $1 \mathrm{GHz}$ bandwidth. The composite signal has a total bit rate of 6 Gbps. A narrowband gap of $300 \mathrm{MHz}$ is inserted to separate the 4-PAM and FBMC signals, and $150 \mathrm{MHz}$ is used to separate the FBMC and UF-OFDM signals. In the converged signal transmission scenario, the BER of $10^{-3}$ can be obtained at a ROP of $-11 \mathrm{dBm}$ for FBMC, at $-9 \mathrm{dBm}$ for UF-OFDM, and $-8 \mathrm{dBm}$ for 4-PAM, after a propagation over $25 \mathrm{~km}$ optical fiber length, between the OLT and the ONU. The 3GPP EVM (\%) limit of $12.5 \%$ is achieved for a ROP of $-9 \mathrm{dBm}$ and $-5 \mathrm{dBm}$ for the 16-QAM FBMC and 16-QAM UF-OFDM, respectively.

For both separate and composite transmission cases, FBMC shows better performance compared to the two other modulation formats 4-PAM and UF-OFDM. 4-PAM will be an attractive candidate compared to conventional OOK for the baseband signal transmission as it doubles the bandwidth efficiency. We believe that the transmission of the converged FBMC, UF-OFDM, and 4-PAM signals in PON will allow $5 \mathrm{G}$ networks to provide high bandwidth to multi-users, while allowing various multiband wireless services.

\section{Acknowledgments}

This work has been carried out within the project FiWiN5G, supported by the European Union's Horizon 2020 research and innovation program. The work of Dr. Eszter Udvary was supported by the BME NC TKP2020 grant of NKFIH Hungary.

\section{References}

[1] SUNG, J. Y., CHOW, C. W., YEH, C. H., et al. Cost-effective mobile backhaul network using existing ODN of PONs for the $5 \mathrm{G}$ wireless systems. IEEE Photonics Journal, 2015, vol. 7, no. 6, p. 1-6. DOI: 10.1109/JPHOT.2015.2497222

[2] SKUBIC, B., FIORANI, M., TOMBAZ, S., et al. Optical transport solutions for $5 \mathrm{G}$ fixed wireless access [Invited]. IEEE/OSA Journal of Optical Communications and Networking, 2017, vol. 9, no. 9 , p. D10-D18. DOI: 10.1364/JOCN.9.000D10

[3] LIU, X., EFFENBERGER, F. Emerging optical access network technologies for $5 \mathrm{G}$ wireless [Invited]. IEEE/OSA Journal of Optical Communications and Networking, 2016, vol. 8, no. 12, p. B70-B79. DOI: 10.1364/JOCN.8.000B70

[4] RAPPAPORT, T. S., SUN, S., MAYZUS, R., et al. Millimeter wave mobile communications for $5 \mathrm{G}$ cellular: It will work! IEEE Access, 2013, vol. 1, p. 335-349. DOI: 10.1109/ACCESS.2013.2260813

[5] TZANAKAKI, A., ANASTASOPOULOS, M., BERBERANA, I., et al. Wireless-optical network convergence: Enabling the $5 \mathrm{G}$ architecture to support operational and end-user services. IEEE Communications Magazine, 2017, vol. 55, no. 10, p. 184-192. DOI: 10.1109/MCOM.2017.1600643

[6] MARTIN, E. P., SHAO, T., VUJICIC, V., et al. 25-Gb/s OFDM $60-\mathrm{GHz}$ radio over fiber system based on a gain switched laser. Journal of Lightwave Technology, 2015, vol. 33, no. 8, p. 1635-1643. DOI: 10.1109/JLT.2015.2391994

[7] SHAMS, H., ZHAO, J. First investigation of fast OFDM radio over fibre system at $60 \mathrm{GHz}$ using direct laser modulation. In Conference on Lasers \& Electro-Optics Europe \& International Quantum Electronics Conference (CLEO EUROPE/IQEC). Munich (Germany), 2013, p. 1-1. DOI: 10.1109/CLEOEIQEC.2013.6801274

[8] BANElli, P., BUZZI, S., COLAVOlPE, G., et al. Modulation formats and waveforms for $5 \mathrm{G}$ networks: Who will be the heir of OFDM? An overview of alternative modulation schemes for improved spectral efficiency. IEEE Signal Processing Magazine, 2014, vol. 31, no. 6, p. 80-93. DOI: 10.1109/MSP.2014.2337391

[9] ZHANG, J. J., XU, M., WANG, J., et al. Full-duplex quasi-gapless carrier aggregation using FBMC in centralized radio-over-fiber heterogeneous networks. Journal of Lightwave Technology, 2017, vol. 35, no. 4, p. 989-996. DOI: 10.1109/JLT.2016.2608138

[10] NGUYEN, T. T., LE, S. T., HE, Q., et al. Multicarrier approaches for high-baudrate optical-fiber transmission systems with a single coherent receiver. IEEE Photonics Journal, 2017, vol. 9, no. 2, p. 1-10. DOI: 10.1109/JPHOT.2017.2672041

[11] XU, M., ZHANG, J., LU, F., et al. FBMC in next-generation mobile fronthaul networks with centralized pre-equalization. IEEE Photonics Technology Letters, 2016, vol. 28, no. 18, p. 1912-1915. DOI: 10.1109/LPT.2016.2575060

[12] BELLANGER, M., LE RUYET, D., ROVIRAS, D., et al. FBMC physical layer: A primer. PHYDYAS, Project Document, Jan. 2010, p. 1-31. [Online] Available at: http://www.ict-phydyas.org/

[13] PARAJUli, H. N., SHAMS, H., GONZALEZ, L., et al. Experimental demonstration of multi-Gbps multi sub-bands 
FBMC transmission in mm-wave radio over a fiber system. Optics Express, 2018, vol. 26, no. 6, p. 7306-7312. DOI: 10.1364/OE.26.007306

[14] SCHAICH, F., WILD, T. Waveform contenders for 5G - OFDM vs. FBMC vs. UFMC. In 6th International Symposium on Communications, Control and Signal Processing (ISCCSP). Athens (Greece), 2014, p. 457-460. DOI: 10.1109/ISCCSP.2014.6877912

[15] WILD, T., SCHAICH, F., CHEN, Y. 5G air interface design based on Universal Filtered (UF-) OFDM. In 19th International Conference on Digital Signal Processing. Hong Kong, 2014, p. 699-704. DOI: 10.1109/ICDSP.2014.6900754

[16] SCHAICH, F., WILD, T., CHEN, Y. Waveform contenders for 5G - Suitability for short packet and low latency transmissions. In IEEE 79th Vehicular Technology Conference (VTC Spring). Seoul (South Korea), 2014, p. 1-5. DOI: 10.1109/VTCSpring.2014.7023145

[17] LAZAROU, I., DRIS, S., BAKOPOULOS, P., et al. Full-duplex 4PAM transmission for capacity upgrade in loop-back PONs. IEEE Photonics Technology Letters, 2013, vol. 25, no. 12, p. 1125-1128. DOI: 10.1109/LPT.2013.2260533

[18] SHIM. H. K., KIM, H., CHUNG, Y. C. 20-Gb/s polar RZ 4-PAM transmission over 20-km SSMF using RSOA and direct detection. IEEE Photonics Technology Letters, 2015, vol. 27, no. 10, p. 1116-1119. DOI: 10.1109/LPT.2015.2408376

[19] STAMATIADIS, C., MATSUMOTO R., YOSHIDA, Y., et al. Full-duplex RSOA-based PONs using 4-PAM with preequalization. IEEE Photonics Technology Letters, 2015, vol. 27, no. 1, p. 73-76. DOI: 10.1109/LPT.2014.2361922

[20] Saljoghei, A., Gutierrez, F. A., Perry, P., et al. Experimental comparison of FBMC and OFDM for multiple access uplink PON. Journal of Lightwave Technology, 2017, vol. 35, no. 9, p. 1595-1604. DOI: 10.1109/JLT.2017.2654319

[21] JUNG, S. Y., JUNG, S. M., HAN, S. K. AMO-FBMC for asynchronous heterogeneous signal integrated optical transmission. IEEE Photonics Technology Letters, 2015, vol. 27, no. 2, p. 133-136. DOI: 10.1109/LPT.2014.2363197

[22] BROWNING, C., FARHANG, A., SALJOGHEI, A., et al. 5G wireless and wired convergence in a passive optical network using UF-OFDM and GFDM. In IEEE International Conference on Communications Workshops (ICCWorkshops). Paris (France), 2017, p. 386-392. DOI: 10.1109/ICCW.2017.7962688

[23] PARAJULI, H. N., UDVARY, E. Wired-wireless converged passive optical network with 4-PAM and multi-sub-bands FBMC. Infocommunications Journal, 2018, vol. 10, no. 2, p. 1-6. ISSN: 2061-2079

[24] PARAJULI, H. N., SHAMS, H., UDVARY, E. Synchronization and channel estimation in experimental M-QAM OFDM radio over fiber systems using CAZAC based training preamble. In
International Conference on Optical Network Design and Modeling (ONDM). Budapest (Hungary), 2017, p. 1-6. DOI: 10.23919/ONDM.2017.7958533

[25] STOHR, A., SHIH, B., ABRAHA, S. T., et al. High spectralefficient 512-QAM-OFDM $60 \mathrm{GHz}$ CRoF system using a coherent photonic mixer (CPX) and an RF envelope detector. In Optical Fiber Communications Conference and Exhibition (OFC). Anaheim (CA, USA), 2016, p. 1-3. ISBN: 978-1-943580-07-1

\section{About the Authors ...}

Hum Nath PARAJULI is a postdoctoral researcher in Sant Anna School of Advanced Studies, TeCIP Institute, Pisa, Italy. He received his B.Eng. degree from Pokhara University, Nepal in 2008 and joint M.Sc. degree from Osaka University, Japan and Scula Superiore Sant' Anna, Italy in 2012 and Ph.D. degree in Electrical Engineering from Budapest University of Technology and Economics, Budapest, Hungary in 2019. His current research interests include photonic integrated circuits design, design of high capacity optical wireless links, millimeter wave communication, digital signal processing for optical communication and design of optoelectronic systems.

Eszter UDVARY received Ph.D. degree in Electrical Engineering from Budapest University of Technology and Economics (BME), Budapest, Hungary, in 2009. She is currently an Associate Professor at BME, Department of Broadband Info-communications and Electromagnetic Theory, where she leads the Optical and Microwave Telecommunication Lab. Dr. Udvary's research interests are in the broad areas of optical communications, include optical and microwave communication systems, Radio over fiber systems, optical and microwave interactions and applications of special electro-optical devices.

Julien POETTE received the B.Sc. degree and the Ph.D. degree in Physics from Rennes-1 University, France, in 2002 and 2005 respectively. He is an Associate Professor at the Grenoble Institute of Technology, France (GrenobleINP) since 2008. His research activities concern optical solutions for radio frequency carrier generation applied to next generation communication systems involving microwave-photonic techniques, and noise studies at millimeter wave frequencies. 